\title{
SCOPE AND LIMITS OF COLOUR MEASUREMENT
}

$\mathrm{A}$

T a Symposium arranged by the Physical Society

Colour Group and held at the Imperial College of Science and Technology, London, on April 2, the present state of progress in the application of toler. ances to colour measurement in widely differing fields was described and discussed. Tolerances represent an important problem in colour control, which the adoption of measurement for the specification of colour has made real and explicit. Papers on colour tolerance in lighting and in colour reproduction also brought in the subjects of colour rendering and tolerances in reproduction.

As it is generally known that standardized colour measurement has been in use for more than a quarter of a century, the onlooker versed in normal techniques of measurement might be excused for thinking that the application of tolerances to measurements of colour is long overdue. But tolerances relate primarily to the quantity measured, and whereas in the measurement of most quantities-by reason of a uniformly valid correlation between differences in the quantity measured and the scale of magnitude adopted-the accuracy or tolerance can be deduced forthwith, in the case of colour this is not so. The magnitudes in terms of which a given colour is 'measured' are to be considered as ultimately linking that colour, through an implied visual judgment of matching equality, with a colour determined by the basis of measurement. The quantity measured thus enters only in individual states of match, like terms in a series, and unrelated with concepts analogous to that of distance, so that no difference relationships exist. A limited range of matching equivalence, determining the effective range of validity of the measurement, is, of course, implied by the existence of a perception limen or threshold. The margin of equivalence is, in a sense, an essential part of the definition of the identity which it is the purpose of any psycho-physical measurement to establish. A measurement of colour is logically not really complete without supplementary information of this character. It lies, moreover, at the root of the problem of colour tolerance.

The information already available on this subject although extensive is not adequate, applying as it does largely to individual observers. The ultimate aim, for the purposes of the psycho-physical and technical applications of colour measurement, would be to obtain data statistically valid and applying to one or more representative sets of conditions of observation. The amount of observational work implied is very great, as may be realized from the fact that the MacAdam single-observer (P.G.N.) (P.G.N. denotes Perley G. Nutting, observer) data ${ }^{1}$ for the chromaticity field at constant luminance for one set of conditions of observation were based upon some 25,000 observations. Priest ${ }^{2}$ in 1928 envisaged the compilation of "comprehensive definitive data" on this subject after the lapse of "a great many years", but this object is still very far from being attained.

Meanwhile, individual methods of applying colour tolerances are not lacking. Eight different formulations of colour tolerance, all of which have their supporters in various applications and laboratories of the world, were described by J. W. Perry in an introductory paper. This remarkable diversity is partly indicative of the fluid state of the subject : a central problem of colour tolerance certainly exists, but its interpretation and possible individual characteristics are different for each industry. The present formulations of colour tolerance depend basically upon actual or approximate discrimination data. Users of MacAdam's ${ }^{3}$ chromaticity ellipses generally incorporate an empirically derived luminance term, although the more complete and elaborate six-term form based upon the work of Brown and MacAdam has also been used. Various alternative systems of colour tolerance based upon different quasi-uniform chromaticity systems have been adopted for use in industry. The most highly developed of these is that due in its original formulation to Judd ${ }^{5}$ and known as the National Bureau of Standards (N.B.S.) system. This permits adjustment of constants to account for super-imposed specular reflexion and for the degree of proximity in the comparison of materials for colour. Various other systems are based upon realizable uniform colour spaces, chief among which is the Munsell system, or upon formulations of functional properties based on these, such as the Saunderson-Milner 'zeta' space. Many colour technologists, concerned as much with the subjective aspects of colour as with formal standardization, base their approach as much upon the Munsell system as upon the International Commission on Illumination (C.I.E.) system.

Although such tolerance systems are used by the leading exponents, there appears to be considerable scope for a more general realization of the value of measurement of colour and assessment of tolerance in industry at large. Thus, much waste of effort results from numerous suppliers attempting to match colours absolutely in the absence of a stated tolerance. Excessive variations in colour occur at times across dyed textiles. An example exhibited by F. L. Warburton (exhibited at the Physical Society Exhibition in March) showed up flagrantly when the limits of variation appeared together at a seam. Architects and others desire to rely upon paint manufacturers in general to adhere to certain stated tolerances (preferably on the Munsell scale). There is clearly some scope for a wider application of colour tolerance.

The varying conditions and requirements applying to different industries were apparent from the discussions. When, as is customary, motor-car body components of appreciable size, made at different times using different batches of paint, are assembled together in a good light, very small differences of colour are perceptible. In this case, as was stated by P. S. Williams, rejections take place if these differences exceed 0.5-1.0 MacAdam units (1942 3 standard-deviation ellipses). This corresponds approximately in colour discrimination to between one-eighth and one-quarter of the difference in colour between the two $D$-lines of sodium, or approximately $0 \cdot 75-1.5$ A. Presumably the economic implications of applying this high standard of rejection are fully realized in the industry, so that parts are not rejected merely because minute differences in colour can be seen in exceptionally favourable circumstances; also that control of colour as a whole, taking into 
account secondary influences such as changes with time, permits of this accuracy. Evidently when economic and technical limitations cease to play an important part in assessment of tolerance, the limits of discrimination of colour become the sole determining factor.

The considerations guiding the Society of Leather Trades' Chemists in formulating specifications of colour tolerance were described by J. S. Mudd. The system adopted is based as regards chromaticity discrimination upon the 'rectangular uniform chromaticity system' of Breckenridge and Schaub, and in respect to luminance upon an adaptation of Nickerson's observations on luminance-factor discrimination. An interesting sidelight on the relative value of instrumentation was provided by the results of tests employing a panel of ten observers It was repeatedly found that near the limit of discrimination, when individual judgments were variable and differed between different observers, a single instrumental test sufficed to give a result agreeing with the average result obtained from ten observers.

In these industries, accelerated fading tests, for which the xenon lamp has been found very serviceable, are based upon measurements of colour-difference referred to such tolerance units. Metameric matches, always undesirable and sometimes prohibitive, call for spectrophotometric as well as colorimetric control and are limited by colour tolerances relating to illuminants of two differing colour-temperatures.

The situation in the colour-printing industry does not appear highly favourable to control of colour in spite of the development of special techniques for increasing the accuracy of colour-printing processes as described by Hughes and Lott. Here it is not the MacAdam ellipses or other unit of visual discrimination which ultimately sets the pace but the accuracy of reproduction of colour by the ink-maker. Matters of cost and other controls necessary in the production of ink apparently limit the colour control possible. Colour tolerances in printing are based entirely upon tests on the inks received by the printer and as a result are often very lax or even impossible of assessment.

Near-white papers are relatively easy to control, as they are mainly subject to a variation of colour in one parameter only, dependent almost entirely upon a varying absorption in the blue region of the spectrum. Bleaching processes and any added coloration may be controlled by abridged spectrophotometry. It was pointed out by J. M. Adams that constant adherence over a long period to a fixed colour specification for a near-white paper is not generally required. White papers are of general interest from the point of view of control of luminance-factor, as it is known that, in estimating whiteness, the eye is able to compensate for a loss of colorimetric purity by an increase in luminance factor. Luminance-factor requirements are known to be very variable in the case of textiles even when purity is high. Quite considerable variations appear admissible in some instances when chromaticity requirements are stringent. Other anomalous cases occur when a tendency to yellowing is resisted in assessment of matching because of implications connected with ageing. In this difficult field the assessment of colour tolerance in measurable terms must rest largely upon specially conducted statistical tests, taking into account all physical and psychological conditions.

From the point of view of standardization of colour tolerance in Britain, the fluorescent lamp industry is fortunate in that it now has virtually no problem, in any event as concerns tolerance upon colour identity. In British Standard 1853 (1956) colour specifications are given for various types of lamps and are accompanied by chromaticity tolerances in terms of MacAdam' ${ }^{3}$ unit discrimination ellipses. (The association with these units of the term 'minimum perceptible colour difference' is unfortunate, as it results in ambiguity and raises questions of interpretation in terms of conditions of perception which are not stated.) Luminance is covered only by minimumflux requirements. It is interesting to learn that fluorescent lamps may readily be produced to a chromatic accuracy comparable with the difference in colour between the two sodium $D$-lines.

A reservation of importance in connexion with the general application of such tolerances is clearly expressed in this standard, namely, that the tolerances stipulated do not include errors of measurement. This is significant in relation to present demands for spectrophotometric accuracy. Such demands, moreover, are not confined to this field. In colorimetry at present, in order to dispense with colour-standards of doubtful permanence, it is required to make absolute measurements of colour to an accuracy exceeding that with which the fine differences of colour can be perceived-much as though an absolutelength interferometer might be required, for everyday use, for measuring lengths of the order of millimetres to a fraction of a wave-length of light. Physical colorimetry is thus confronted with demands for purposes of routine measurements far more exacting than any which could be met in the laboratory without recourse to interferometry, no counterpart of which as a powerful auxiliary to metrology exists for photometry.

Maintaining a standard chromaticity for the colour appearance of a fluorescent lamp still leaves wide scope for variation in the distribution of spectral energy which, if not suitably controlled, can seriously affect its acceptance as a general-purpose illuminant. Assume, for example, $n$ given spectral reflexion functions relating respectively to $n$ different, familiar, distinctively coloured objects and giving rise, in conjunction with a certain, approximately black-body illuminant, to $n$ different chromaticities and corresponding colour sensations under certain ordinary conditions. Then between these different sensations, when simultaneously perceived as associated with the respective objects, a complex subjective relation exists, related to the observing conditions and attributable to an acquired sense of chromatic morphology in the field of colour recognition. This relation is readily appreciable (although at present not precisely definable) and, by virtue of experience and adaptation, is fairly stable for a change in energy distribution in the range of near-black-body illuminants. The widely differing energy distributions possible in the mixed line spectrum and continuum of the fluorescent lamp spectrum are apt, however, to disturb this interchromatic relation unduly unless careful control is exercised. Again we are concerned with determining in physical terms the range of validity of a subjective colour relation.

The simple control of the energy distribution in relative terms applying to specified spectrum bands, which is the method most generally favoured, is the subject of much current discussion and long-standing investigation. Dr. J. W. Strange emphasized the inappropriateness, in his view, of applying more exacting tolerances to spectral energy distribution 
than are justified by the available results of experimental test, and favoured a five-band division of the spectrum for the control of energy distribution $\rightarrow$ one of the methods included in British Standard 1853 (1956).

Colour photography, with its possibilities for documentary and scientific record as well as for pictorial representation, was discussed as regards accuracy from both aspects : it is well known, however, that bearing in mind the inherent limitations of the process, its local colour accuracy is not to be reckoned in terms of discrimination limens. Dr. R. W. G. Hunt presented a tentative theory of colour tolerance for pictorial similitude under varying conditions of adaptation which, subject to the validity of some doubtful assumptions, appeared to provide the basis for a theoretical criterion for the further refinement of the process.

J. W. Perry

MacAdam, D. X., J. Opt. Soc. Amer., 32, 247 (1942).

2 Priest, I. G., J. Opt. Soc. Amer., 16, 117 (1928).

${ }^{3}$ MacAdam, D. L., J. Opt. Soc. Amer., 33, 18 (1943).

Brown, W. R. J., and MacAdam, D. L., J. Opt. Soc. Amer., 39, 454 (1949).

'Judd, D. B., Amer. J. Physiol., 52, $418(1939)$. See also Scofield, F., Judd, $D_{\text {. B ., and Hunter, }}$ R. S., Bull. Amer. Soc. Test. Mat., (1942).

\section{GENETIC ASPECT OF SOMATIC CELL VARIATION}

W HILE our knowledge of variation within populations of micro-organisms is large and impressive, technical difficulties have impeded advance in the study of cell variation in somatic tissues. In consequence, our understanding of the basic feature in organic development and differentiation is scanty. It was therefore timely to choose the genetical aspects of somatic cell variation as the topic of discussion for the eleventh Biology Research Conference, held in Gatlinburg, Tennessee, during April 2-5, sponsored by the Biology Division of the Oak Ridge National Laboratory. About two hundred and fifty scientists attended.

The wide spectrum of somatic cell variations attributable to changes in the nuclear or cytoplasmic determinants of heredity was reviewed by C. Stern (Berkeley) and B. Ephrussi (Paris). In these detailed surveys, examples were given of various nuclear phenomena such as somatic crossing-over, rnutation, genome separation, chromosome elimination, nuclear fusion, and endomitosis which result in cellular variation. Ephrussi described particular changes occurring in cytoplasmic components, such as plastids and microsomes, and which can be studied by genetical methods.

Cells vary in their capacity to produce specific proteins which behave as antibodies. A. H. Coons (Boston) pointed out that cells not only acquire the ability to manufacture antibodies, but also retain this ability long after the antigenic stimulus has been removed. Coons provided evidence to show that these particular cells are stimulated to proliferate by a second exposure to antigen. The new cell population has the same immunological response. This phenomenon has all the characteristics of acquired inheritance of a cellular function. While Coons used immunological reagents tagged with fluorescent dyes to study antibody formation in vivo, J. Lederberg (Madison) and E. S. Lennox (Urbana) studied antibody production by single cells from hyperimmune animals in vitro. All these investigators failed to find evidence of production of more than one antibody by a single cell at any one time.

C. W. Cotterman (Dallas) analysed several cases of irregularity in the human ABO blood group in which a minute proportion of blood cells was of aberrant antigenic type. K. C. Atwood (Oak Ridge) described a method by which phenotype variability in human erythrocytes can be detected. Some of the variations discussed by these investigators may be explainable only by mutation, some may be serological freaks, others are no doubt only cases of homozygosis for one of a pair of heterozygous gene loci, brought about by loss, non-disjunction or somatic crossingover of chromosomes. Though the exact mechanism by which Atwood's exceptional cells arise is yet to be determined, it seems reasonable to assume that in some instances mutation is the cause. Atwood estimates that the mutation-rate in the human erythrocyte is $3 \times 10^{-9}$ per hour or $7 \times 10^{-6}$ per cell division.

The antigenic properties of tumour cells have been studied by G. Klein (Stockholm). He induced tumours in $F_{2}$ hybrids of coisogenic resistant mice heterozygous for $H$-2 histocompatibility locus, and changes were detected in antigenicity when they were transplanted into the parental strains. The new tumour variants of Klein showed predilection for one of the parental strains, which raises the intriguing question : Does the missing complementary variant represent a reversion to normal cell behaviour ? J. Lederberg (Madison) in his summing-up of the Conference proposed the idea that Klein might be dealing with homozygosis for one of the two $H$-2 alleles in the heterozygote due to somatic crossing-over and that the closely linked and dominant gene for malignancy has been lost from one of the sister cells.

How specific interaction of genes may be the source of cellular variation was discussed by R. A. Brink (Madison). Analysing the behaviour of a mutable gene which determines pericarp colour in the endosperm of maize, he was able to disentangle some of the complexity of interaction between gene and its 'controllers' or 'modulators'. The latter are spatially associated with the gene and when they are transposed to new loci, phenotype differences are produced. Brink found no correlation of 'modulator' activity with orderly development, which suggests that they are not important determinants of differentiation. The possible path of interaction between factors, which results in the formation of the melanotic pseudo-tumours in Drosophila, was discussed by C. Barigozzi (Milan).

Impressive methodical refinements, both cytological and serological in nature, were employed by T. S. Hauschka (Buffalo) to demonstrate the wide spectrum of heterogeneity in tumour cell populations, and the various degrees of correlation which may exist between particular properties, for example, antigenic specificity, drug resistance and chromosome number. C. E. Ford (Harwell) carried out cytological analysis of spontaneous primary tumours in mice and those induced by radiation, and found that in some tumours the chromosome number was the same 